Title

\title{
Molecular recognition: how photosynthesis anchors the mobile antenna
}

\section{Authors}

John F. Allen

Research Department of Genetics, Evolution and Environment, Darwin Building, University

College London, Gower Street, London, United Kingdom, WC1E 6BT

E-mail: j.f.allen@ucl.ac.uk

ORCID: 0000-0002-0950-0429

Jon Nield

School of Biological and Chemical Sciences, Queen Mary University of London, Mile End

Road, London, United Kingdom, E1 4NS

E-mail: j.nield@qmul.ac.uk

ORCID: 0000-0001-9983-0239

Norbert Krauß

Karlsruher Institut für Technologie (KIT), Botanisches Institut, Fritz-Haber-Weg 4, Gbd.

30.43, D-76131 Karlsruhe, Germany

E-mail: norbert.krauss@kit.edu

ORCID: 0000-0002-7128-4632

\begin{abstract}
True to its name, Light Harvesting Complex II (LHC II) harvests light energy for photosystem II. But LHC II can stray, harvesting light energy for photosystem I instead. Cryo-electron microscopy now shows how this mobile antenna becomes so attached to its new partner.
\end{abstract}

\section{Key Words}


Photosynthesis, Light Harvesting Complex II, Photosystem I, Photosystem II, Light State Transitions, Chloroplast Thylakoid

\section{Text}

\section{Harnessing Sunlight}

Light-Harvesting Complex II (LHC II) is a membrane protein that contains chlorophyll and carotenoid pigment molecules. The chlorophylls absorb light and transfer its energy to the photochemical reaction centre of chloroplast photosystem II. In the reaction centre itself, the absorbed excitation energy oxidises a specific chlorophyll molecule. This primary reaction initiates electron transport in photosynthesis. The oxidised chlorophyll is re-reduced with electrons supplied from the oxidation of water, yielding free molecular oxygen - upon which all aerobic life depends. The electrons from photosystem II (PS II) arrive eventually at photosystem I (PS I), where absorbed light energy is harnessed in a similar way, by chlorophyll oxidation, but in a separate and distinct reaction centre. PS II and PS I are therefore connected in series, which means that their electron currents are equal, as depicted in the Z-scheme of non-cyclic photosynthetic electron transport. PS I can also pass electrons back to itself, in a cyclic pathway that is also coupled to ATP synthesis, but without the direct need for an initial electron donor or terminal electron acceptor. Working together in this way, photosystems I and II harness sunlight for carbon and nitrogen assimilation, providing all of our food and most of our fuel.

Some individual LHC II pigment-protein complexes are able to collect light energy either for PS I or for PS II. For optimal efficiency, the rates of absorbed excitation energy transfer to the two reactions centres must match their rates of electron transfer. Redirection and redistribution of LHC II between PS II and PS I achieve this effect. Phosphorylation of a sidechain of one of its polypeptides redirects LHC II to PS I; dephosphorylation returns it to PS II. This reversible, post-translational modification of LHC II produces balanced excitation energy distribution between the two photosystems of green plants and algae.

How does LHC II switch its antenna function between PS II and PS I? Protein phosphorylation in LHC II causes it to bind to PS I. A cryo-EM structure for the resulting supercomplex [1] now shows how the phosphoryl group stabilises a protein secondary structure that anchors photosystem I and LHC II together. 


\section{The Busy Life of LHC II}

Pan et al. present a new model at 3.3 A resolution of the maize (Zea mays) pLHC II-PS I supercomplex that is formed when leaves are exposed to orange light, or light 2 , which initially favours PS II (Figure 1) [1]. This state of adaptation to light 2 is light-state 2, or just "state 2" - in which a fraction of absorbed light energy has become redistributed to PS I at the expense of PS II [2]. LHC II is a trimer. Each component polypeptide (termed Lhcb1, Lhcb2, etc.) has three transmembrane helices and binds 14 chlorophyll molecules $[3,4]$. The new model shows one specific LHC II polypeptide, Lhcb2, in its phosphorylated form (pLhcb2), closely aligned with the PS I reaction centre complex (Figure 1A). pLhcb2 adopts a small, stable, compact structure at and around the phosphorylation site, which is a threonine residue close to its amino terminus. Two basic amino acid residues near the $\mathrm{N}$-terminus of pLhcb2 point away from the threonine phosphorylation site (Figure 1B). Of these, Arg1 "folds back" (away from PS I) to stabilise the phosphate group by an ionic interaction. By forming a salt bridge (with Glu67) and a hydrogen bond with the Ser72 side chain, the Arg2 side chain in pLHC II-PS I plays a role in stabilising the interface of pLHC II with the PS I reaction centre polypeptide PsaL.

Pan et al. looked for the degree of conservation of the interface of PsaL polypeptide of PS I with pLhcb2, and assumed Lhcb2 to be the phosphorylated subunit of LHC II that interacts with the PS I core, as indicated by biochemical studies with chloroplasts from Arabidopsis thaliana [5].

In the maize cryo-EM structure, the N-terminal portion of Lhcb2 looks like a rope with a grappling hook (containing the phosphate group) that has been thrown at the PS I core to become fixed into a binding pocket on the stromal side of PS I (Figure 1A). There seems to be no precedent for a structured, folded, but "free-floating" polypeptide segment such as that suggested from residues 4 to 8 of the Lhcb2 N-terminus, which adopts a defined threedimensional structure, but is not packed against another segment of polypeptide or any other molecule resolved in the structure.

\section{Open Questions}

A key point from these structural studies is that phosphorylation induces a change in structure that tips the affinity of LHC II in favour of PS I, at the expense of PS II. But questions remain. How large is the change in structure? What are the default contacts between LHC II and PS II, and how and why are these overridden? Is there an extensive structural change in 
LHC II upon phosphorylation, or does the N-terminus of pLhcb2 just form something resembling a hook on the end of a piece of rope? A piece of rope is flexible, and this analogy is not obviously reconciled with precise orientation, a docking event, that might be required for efficient excitation energy transfer between chlorophylls of pLHC II and PS I. Can the "rope" shorten or wind?

The structural model for pLHC II-PS I [1] has implications concerning chloroplast thylakoid membrane lateral heterogeneity with its separation of PS I and PS II between different membrane domains. The LHC II-donor, PS II, and the LHC II-acceptor, PS I, can be in close proximity, most likely in the grana margin or "end membrane" [6]. This raises again questions concerning lateral migration of LHC II, and suggests that phospho-LHC II does not need to undertake any long, solitary journey from chloroplast thylakoid grana membranes (stacked) to stroma membranes (unstacked) during the transition state 2. A local, phosphorylation-induced anchor or grappling hook thrown out from LHC II could alter interactions of closely adjacent proteins in such a way as to alter membrane topology and thylakoid membrane stacking. Thus a small, local, secondary structure could be viewed as a new recognition surface or as a 'plug-in' module for insertion and reattachment of phosphoLHC II to PS I. Initiation of a global conformational change might create a docking surface and a variety of contact points for functional interaction with polypeptides on PS I. PS I itself might undergo a conformational change to allow its own $\mathrm{PsaL} / \mathrm{H} / \mathrm{O}$ surface region to become more amenable to docking by pLHC II.

PS I from pea (Pisum sativum) chloroplasts is monomeric, unlike the oligomeric PS I of cyanobacteria [7]. Oligomer formation in plant PS I may be prohibited by the presence of a single-helix subunit not found in cyanobacteria; PsaH. Mutational analysis of PsaH suggests that it is required for binding PS I to phospho-LHC II in state 2. This binding requires additional small photosystem I subunits, including a $4 \mathrm{kDa}$ protein termed PsaI together with PsaL. The plant PS I complex contains four monomeric LHC I (Lhca) subunits in a "crescent moon" configuration [8], each similar in overall structure to one Lhcb subunit of trimeric LHC II. The binding affinity of PsaI/H/L for phospho-LHC II might also increase on the lumen-exposed side of the membrane [9], even though the phosphorylation site itself is on the stromal side.

Both the exact location of the $\mathrm{N}$-terminal phosphorylation site and the polypeptide composition of LHC II oligomers differ between pea, spinach (Spinacia oleracea), arabidopsis (Arabidopsis thaliana) and chlamydomonas (Chlamydomonas reinhardtii). It 
seems likely that the composition of the phospho-LHC II-PS I supercomplex is speciesspecific. Across green plant species and phyla, how general is the anchoring mechanism revealed by Pan et al. for maize (Zea mays)?

\section{Implications and future directions}

Structures of arabidopsis chloroplast PS I with LHC II in light-state 2 have been obtained by single particle transmission EM [5, 10], and show electron densities that correspond to those of the LHC II trimers resolved by X-ray crystallography. In agreement with the cryo-EM structure of Pan et al. [1], the phospho-LHC II trimer attaches to the periphery of PS I at a site opposite to that occupied by the four monomeric LHC I (Lhca) components. This LHC II binding site (see Box 1 and Fig. 1B) is occupied by PsaH, PsaO and PsaL, mutant plants of which are impaired in the ability to make the transition to light state 2. PsaI has an indirect effect by stabilising the interface. Single particle EM also shows smaller electron densities attached to photosystem I in state 2 [11], and these are suggested to correspond to CP29 or to monomeric LHC II. The presence of a protonmotive photosynthetic complex I $[12,13]$ in an arabidopsis photosystem I supercomplex $[10,11]$ is consistent with light-state 2 supporting increased cyclic electron transport and photophosphorylation.

From the cryo-EM structural studies of Pan et al. [1], there can be little doubt that phosphorylated LHC II detaches from PS II and binds to PS I, thus accounting for the welldocumented increase in PS I light-harvesting antenna size, and decrease in PS II antenna size, in light state 2 [2]. The mechanism underlying this transition involves forces operating over atomic distances - effects on membrane interactions, and on photosystem distribution between membrane domains, are consequences rather than causes. Phosphorylation induces a local change of secondary structure in LHC II that entails tertiary structural changes and perhaps quaternary structural changes with effects on the reaction centre cores of PS I and PS II.

Protein structural description of the mechanism of state 1-state 2 transitions has been a goal for decades [2]. The findings of Pan et al. [1] outline the molecular basis of these adaptations of the photosynthetic apparatus. Future structural investigation can now focus usefully on conformational changes involved in molecular recognition guided by reversible phosphorylation of both LHC II and PS II reaction centre proteins [14], as well as on other post-translational modifications that regulate photosynthesis [15]. 


\section{Acknowledgements}

JFA thanks the Leverhulme Trust for Emeritus Research Fellowship EM-2015-068. JN is grateful for the support of the Japan Science and Technology Agency CREST program JPMJCR13M4. NK thanks the Deutsche Forschungsgemeinschaft (DFG) for research grant KR-2034/1-1.

\section{References}

1 Pan, X., et al. (2018) Structure of the maize photosystem I supercomplex with lightharvesting complexes I and II. Science 360, 1109-1113

2 Allen, J.F. (2017) Why we need to know the structure of phosphorylated chloroplast lightharvesting complex II. Physiologia Plantarum 161, 28-44

3 Liu, Z., et al. (2004) Crystal structure of spinach major light-harvesting complex at $2.72 \AA$ resolution. Nature 428, 287-292

4 Standfuss, J., et al. (2005) Mechanisms of photoprotection and nonphotochemical quenching in pea light-harvesting complex at 2.5 A resolution. EMBO J 24, 919-928 5 Crepin, A. and Caffarri, S. (2018) Functions and Evolution of Lhcb Isoforms Composing LHCII, the Major Light Harvesting Complex of Photosystem II of Green Eukaryotic Organisms. Current Protein \& Peptide Science 19, 699-713

6 Koochak, H., et al. (2019) The structural and functional domains of plant thylakoid membranes. The Plant Journal 97, 412-429

7 Jordan, P., et al. (2001) Three-dimensional structure of cyanobacterial photosystem I at 2.5 Å resolution. Nature 411, 909-917

8 Mazor, Y., et al. (2015) The structure of plant photosystem I super-complex at $2.8 \AA$ resolution. eLife 4, e07433

9 Ding, J.H., et al. (2014) The impact of N-terminal phosphorylation on LHCII conformation in state transition. Acta Mechanica Sinica 30, 447-456

10 Yadav, K.N.S., et al. (2017) Supercomplexes of plant photosystem I with cytochrome b6f, light-harvesting complex II and NDH. Biochimica et Biophysica Acta (BBA) - Bioenergetics $1858,12-20$ 
11 Kouřil, R., et al. (2018) Organization of Plant Photosystem II and Photosystem I

Supercomplexes. In Membrane Protein Complexes: Structure and Function (Harris, J.R. and Boekema, E.J., eds), pp. 259-286, Springer Singapore

12 Laughlin, T.G., et al. (2019) Structure of the complex I-like molecule NDH of oxygenic photosynthesis. Nature 566, 411-414

13 Schuller, J.M., et al. (2019) Structural adaptations of photosynthetic complex I enable ferredoxin-dependent electron transfer. Science 363, 257-260

14 Liu, X.Y., et al. (2019) Structural Insights into Substrate Selectivity, Catalytic Mechanism, and Redox Regulation of Rice Photosystem II Core Phosphatase. Molecular Plant 12, 86-98

15 Koskela, M.M., et al. (2018) Chloroplast Acetyltransferase NSI Is Required for State Transitions in Arabidopsis thaliana. Plant Cell 30, 1695-1709

\section{Figure 1.}

\section{Figure 1A. A structural model for the maize chloroplast supercomplex containing} photosystem I bound to phosphorylated LHC II. The view is within and parallel to the horizontal thylakoid membrane plane, with the stromal surface to the top. The supercomplex is formed in light state 2. The path of electron transfer within the PS I reaction centre is vertically upward, from lumen to stroma. Light energy is absorbed and transferred by chlorophyll $a$ (dark green), chlorophyll $b$ (light green) and by carotenoids (orange), all rendered as sticks. Individual polypeptides are depicted as ribbons coloured arbitrarily to distinguish between them, though the single, phosphorylated LHC II polypeptide, pLhcb2, is shown in yellow. CPK-coloured spheres and sticks show residues 1 to 3 of LHC IIb and the Fe-S electron acceptors on the stromal side of the PS I reaction centre. Photosystem I is shown in surface representation.

Figure 1B. The PS I binding pocket for the LHC II polypeptide pLhcb2. Residues of the photosystem I core that form hydrogen bonds with pLhcb2 are shown as sticks, those which are only in van der Waals contact with pLhcb2 as sticks and spheres. For pLhcb2 only residues 1-3 are shown as sticks, the remaining portion as a cartoon. Residues of PsaH are shown in blue, those of PsaL in green, and Arg39 of PsaO in magenta. Black dashed lines 
indicate potential hydrogen bonds. The intrapolypeptide ionic interaction between Arg1 and pThr3 that stabilises the N-terminal structure of pLhcb2 is shown by grey dashed lines.

Graphics prepared from 5zji.pdb [1] using the program PyMOL (The PyMOL Molecular Graphics System, Version 2.1 Schrödinger, LLC, see: https://pymol.org).

\section{Box 1}

\section{Intimate Contact - At the Interface}

In the cryo-EM-based structure of Pan et al. (Figure 1B), PsaH and PsaL bind phospho-LHC II, but PsaO is also part of the binding interface. PsaI does not bind directly to phospho-LHC II, but stabilises PsaH and thereby the LHC II docking site. Furthermore, no LHC I subunit is involved in binding of phospho-LHC II, and the anchoring of phospho-Lhcb2 on PS I takes place entirely on the stromal side. There are, nevertheless, several van der Waals contacts between LHC II and PS I in the membrane-intrinsic region of the holocomplex. On the LHC II side these contacts are formed mainly by chlorophylls and carotenoids. It is likely that further stabilisation of the interface in this region is achieved by lipid molecules that are unresolved in the electron density map. Taken together, these observations mean that the stability of the phospho-LHC II-PS I complex is determined by the membrane-intrinsic hydrophobic interactions in combination with the interactions of surface-exposed segments on the stromal side of the membrane. Only the directed bonds that Pan et al. found within the stromal part of the interface - a salt bridge and hydrogen bonds - are suitable to restrain pLHC II in a defined position and orientation with respect to PS I.

In arabidopsis the LHC II L-trimer ('L' for 'loosely-bound' to PS II) contains Lhcb1 and Lhcb2 chains and forms part of the antenna of PS I when Lhcb2 becomes phosphorylated [5]. Crepin and Caffarri [5] conclude that phosphorylation of Lhcb2 alone is sufficient for formation of the LHC II-PS I supercomplex; the binding site of LHC II with PS I involves a direct interaction of the Lhcb2 phosphothreonine with PsaH. This conclusion fits closely with the structure described by Pan et al., where PsaH is in van der Waals and hydrogenbonding contact with the N-terminal region of phospho-Lhcb2, while the structure described by Pan et al. reveals no direct interaction of PsaH with the phosphothreonine itself. 